\title{
Dielectric Properties of Poly- and Single-Crystalline $\mathrm{BaTi}_{2} \mathrm{O}_{5}$
}

\author{
Rong Tu and Takashi Goto \\ Institute for Materials Research, Tohoku University, Sendai, Japan 980-8577
}

Non-oriented and $(010)$ oriented poly-crystalline $\mathrm{BaTi}_{2} \mathrm{O}_{5}\left(\mathrm{BT}_{2}\right)$ was prepared by hot-pressing and arc-melting, respectively. The dielectric properties of poly-crystalline $\mathrm{BT}_{2}$ were compared with those of single-crystalline $\mathrm{BT}_{2}$ prepared by a floating zone method. The maximum permittivity of (010) oriented poly-crystalline $\mathrm{BT}_{2}$ was 2000 at $720 \mathrm{~K}$, whereas that of single-crystalline $\mathrm{BT}_{2}$ was 6000 at the Curie temperature, $T_{\mathrm{c}}(750 \mathrm{~K})$. The permittivity of non-oriented poly-crystalline $\mathrm{BT}_{2}$ was 30 to 300 , having a small peak at $720 \mathrm{~K}$. The tan $\delta$ of all specimens was 0.01 to 0.2 below $T_{\mathrm{c}}$ and significantly increased with increasing temperature above $T_{\mathrm{c}}$. The electrical conductivity of (010) oriented poly- and single-crystalline $\mathrm{BT}_{2}$ had almost same values, which were one order higher than that of non-oriented poly-crystalline $\mathrm{BT}_{2}$ and (001) oriented single-crystalline $\mathrm{BT}_{2}$. [doi:10.2320/matertrans.47.2898]

(Received May 31, 2006; Accepted October 13, 2006; Published December 15, 2006)

Keywords: $\mathrm{BaTi}_{2} \mathrm{O}_{5}$, poly-crystalline, single-crystalline, arc-melting, hot-pressing, floating zone method, permittivity, Curie temperature

\section{Introduction}

Although the phase diagram of $\mathrm{BaO}-\mathrm{TiO}_{2}$ system has been studied for a long time, the ferroelectricity of $\mathrm{BaTi}_{2} \mathrm{O}_{5}\left(\mathrm{BT}_{2}\right)$ has not been known until recently. ${ }^{1-5)}$ Our group ${ }^{6,7)}$ and Akishige et $a l .{ }^{8)}$ independently synthesized single-crystalline $\mathrm{BT}_{2}$ and reported the significant ferroelectricity only in the $b$ direction. Since $\mathrm{BT}_{2}$ can be easily decomposed into $\mathrm{BaTiO}_{3}$ (BT) and $\mathrm{Ba}_{6} \mathrm{Ti}_{17} \mathrm{O}_{40}\left(\mathrm{~B}_{6} \mathrm{~T}_{17}\right)$ above $1500 \mathrm{~K}$, large-scale crystalline $\mathrm{BT}_{2}$ can be hardly prepared. In order to apply $\mathrm{BT}_{2}$ for practical applications, the process to prepare $b$-axis oriented poly-crystalline $\mathrm{BT}_{2}$ should be developed. Although the conventional solid state sintering process may not be applicable due to the decomposition of $\mathrm{BT}_{2}$, Beltrán et al. have reported the preparation of non-oriented poly-crystalline $\mathrm{BT}_{2}$ in a single phase by a sol-gel method, in which the $T_{\mathrm{c}}$ was about $750 \mathrm{~K}$ with the permittivity of $130 .{ }^{9)}$ It is known that needle-like single-crystalline $\mathrm{BT}_{2}$ would precipitate from melts, where the growth direction is parallel to $b$-axis. ${ }^{8)}$ This suggests that the $b$-axis oriented poly-crystalline $\mathrm{BT}_{2}$ can be prepared by a melt-solidification process. In the present study, an arc-melting method was applied to prepare the $b$-axis oriented poly-crystalline $\mathrm{BT}_{2}$, and the dielectric properties were compared with those of non-oriented polyand single-crystalline $\mathrm{BT}_{2}$.

\section{Experimental}

Dried $\mathrm{BaCO}_{3}$ and $\mathrm{TiO}_{2}$ powders (purity: 99.9\%) were weighed and mixed at the molar ratio of 1 to 2 , and pressed into pellets $(20 \mathrm{~mm}$ in dia., $5 \mathrm{~mm}$ in thick). The pellets were calcined at $1173 \mathrm{~K}$ in air for $43 \mathrm{ks}$ and then melted with an arc using a tungsten electrode. The arc-melting apparatus was evacuated to $10^{-3} \mathrm{~Pa}$ and $\mathrm{Ar}$ gas was introduced into the chamber at $2.5 \mathrm{kPa}$. Button specimens were obtained by melting the pellets, which were quenched on a water-cooled copper plate, and were cut to coupons $(5 \times 5 \times 1 \mathrm{~mm})$ whose surface is parallel to the copper plate. The coupons were heattreated at $1223 \mathrm{~K}$ for $43 \mathrm{ks}$ in air. The button specimens were also crushed into powder. The powder was hot-pressed at $1473 \mathrm{~K}$ under $30 \mathrm{MPa}$ for $43 \mathrm{ks}$ in $\mathrm{O}_{2}$ in an alumina die, and sintered bodies $(10 \mathrm{~mm}$ in dia, $1 \mathrm{~mm}$ in thick) were obtained.
A floating zone (FZ) method was used to prepare singlecrystalline $\mathrm{BT}_{2}$ whose surface were cut parallel to (001) and (010) planes $^{6)}$ in size of $2 \times 2 \times 0.3 \mathrm{~mm}$. The crystal phase was identified by X-ray diffraction (XRD). The microstructure was observed by scanning electron microscope (SEM) after etching in an $\mathrm{HCl}+10 \mathrm{vol} \% \mathrm{HF}$ solution at room temperature. The composition was analyzed by electron probe microanalysis (EPMA). A gold paste was used as electrodes. The dielectric properties were measured in air with an impedance analysis (Hewlett Packard 4194) from 293 to $1073 \mathrm{~K}$ in a frequency $(f)$ range between $10^{2}$ and $10^{7} \mathrm{~Hz}$. The DC electrical conductivity was measured with a 2-probe method in air.

\section{Results and Discussion}

Figure 1 shows the XRD patterns of poly- and singlecrystalline $\mathrm{BT}_{2}$. The poly-crystalline $\mathrm{BT}_{2}$ prepared by hotpressing was in a single phase with almost no preferred orientation (Fig. 1(a), abbreviated as HP). The arc-melted poly-crystalline $\mathrm{BT}_{2}$ (Fig. 1(b) as AR) had the significant (010) orientation. The single-crystalline $\mathrm{BT}_{2}$ specimens

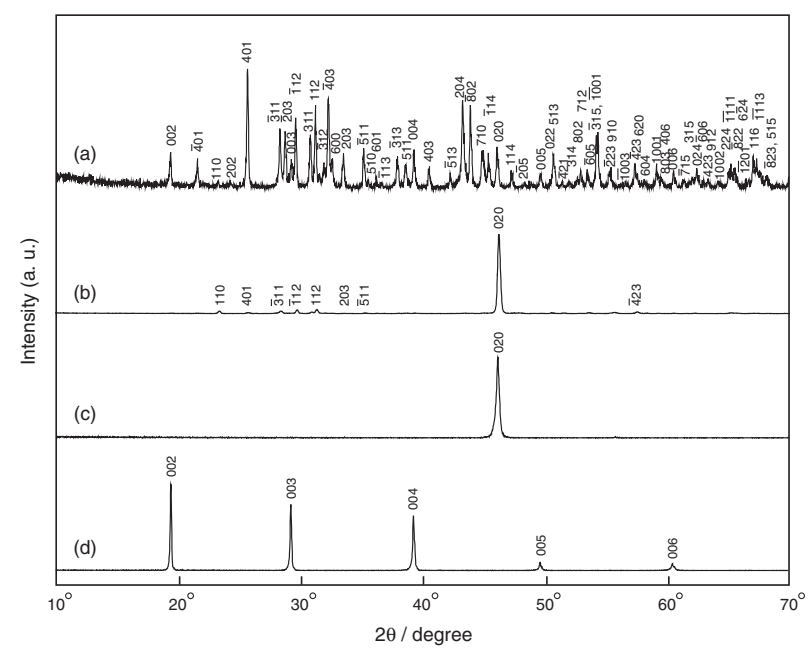

Fig. 1 X-ray diffraction patterns of poly- and single-crystalline $\mathrm{BT}_{2}$ (a) HP, (b) AR, (c) $b$-FZ and (d) $c$-FZ. 

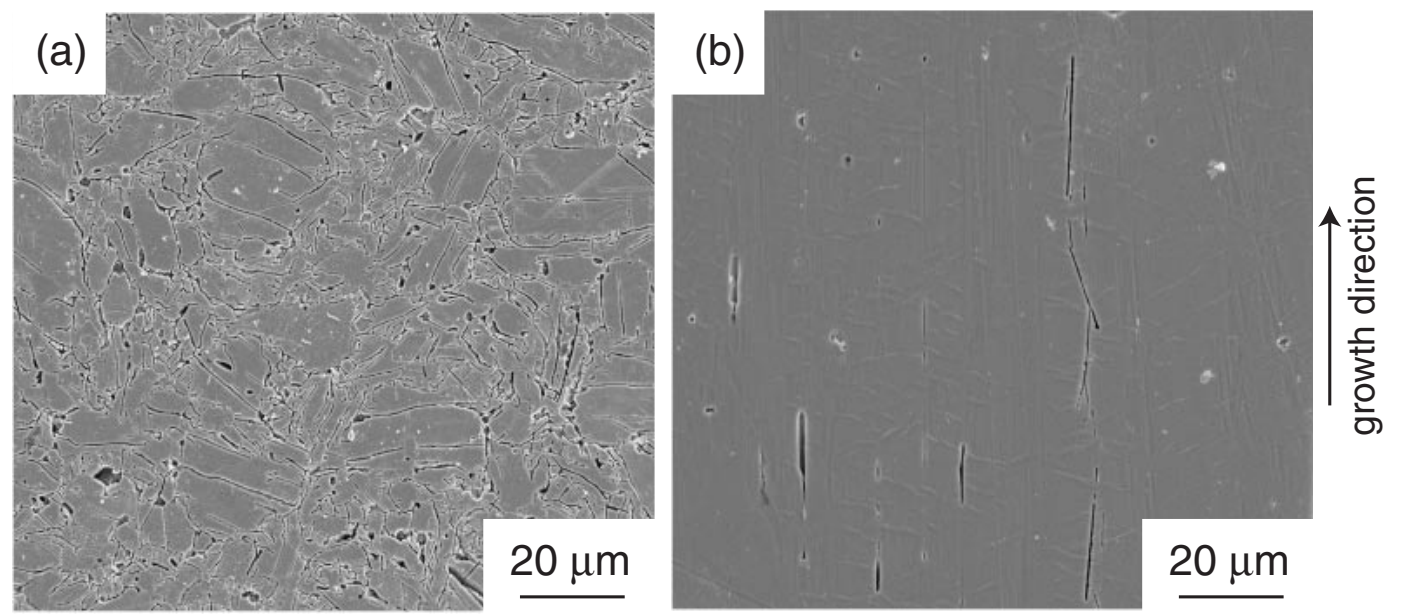

Fig. 2 SEM images of cross-section for poly-crystalline BT $\mathrm{BT}_{2}$ (a) HP and (b) AR paralleled to growth direction.

whose (010) and (001) planes were parallel to the surface are abbreviated as $b$-FZ and $c$-FZ in Fig. 1, respectively.

Figure 2 depicts the SEM images of cross-section for HP and AR specimens parallel to the growth direction. Elongated grains were observed in the HP specimens (Fig. 2(a)). It is well known that $\mathrm{BT}_{2}$ crystal may easily grow along the $b$ direction from the melt and needle-like $\mathrm{BT}_{2}$ crystals were often prepared. ${ }^{8}{ }^{80}$ Although no report on the abnormal grain growth during the sintering of $\mathrm{BT}_{2}$, the elongated direction might be the $b$-axis of $\mathrm{BT}_{2}$. Many gaps at the grain boundaries might be formed by significant difference of the linear thermal expansion $(\alpha)$ among $a$-, $b$ - and $c$-directions of $\mathrm{BT}_{2}\left(\alpha_{a}=5.14, \alpha_{b}=0.86\right.$ and $\alpha_{c}=12.5 \times 10^{-6} \mathrm{~K}^{-1}$ at $\left.900 \mathrm{~K}^{10)}\right)$. The AR specimens were dense having a columnar texture along the growth direction (Fig. 2(b)). Since XRD data (Fig. 1(b)) showed significant (010) orientation, the growth direction of the columnar grains should be $\langle 010\rangle$. Asmelted AR specimens were dark blue which changed to white in color after heat-treatment in air, suggesting the compensation of oxide vacancy in the as-melted AR specimens. Since $b$-FZ and $c$-FZ specimens were dense with no specific feature, no SEM images were presented.

Figure 3 demonstrates the complex impedance plots at 743 and $973 \mathrm{~K}$. At $743 \mathrm{~K}$, the impedance of all specimens was too high to draw full semicircles at the present frequency range. The magnified view close to the original point was inserted in Fig. 3(a). The impedance of AR specimens was almost the same as that of $b$-FZ because both specimens were highly $b$ axis oriented. The HP specimen had intermediate value between $b$-FZ and $c$-FZ specimens consisting with the nonorientation. At $973 \mathrm{~K}$, all specimens showed full semicircles crossing the original point. The capacitance values associated to the semicircle can be calculated at the top of semicircle from the relationship of $\omega \tau=1$, where $\omega(=2 \pi f)$ is an angular frequency and $\tau$ ( $=R C, R$ : resistivity, $C$ : capacitance) is a relaxation time. The associated capacitance values were $30,60,40$ and $7 \mathrm{pF}$ for $\mathrm{AR}, \mathrm{HP}, b$-FZ and $c$-FZ specimens, respectively. These values could be caused of the bulk response, and no grain boundary response was identified in the poly-crystalline AR and HP specimens suggesting no specific grain boundary phase. ${ }^{11)}$
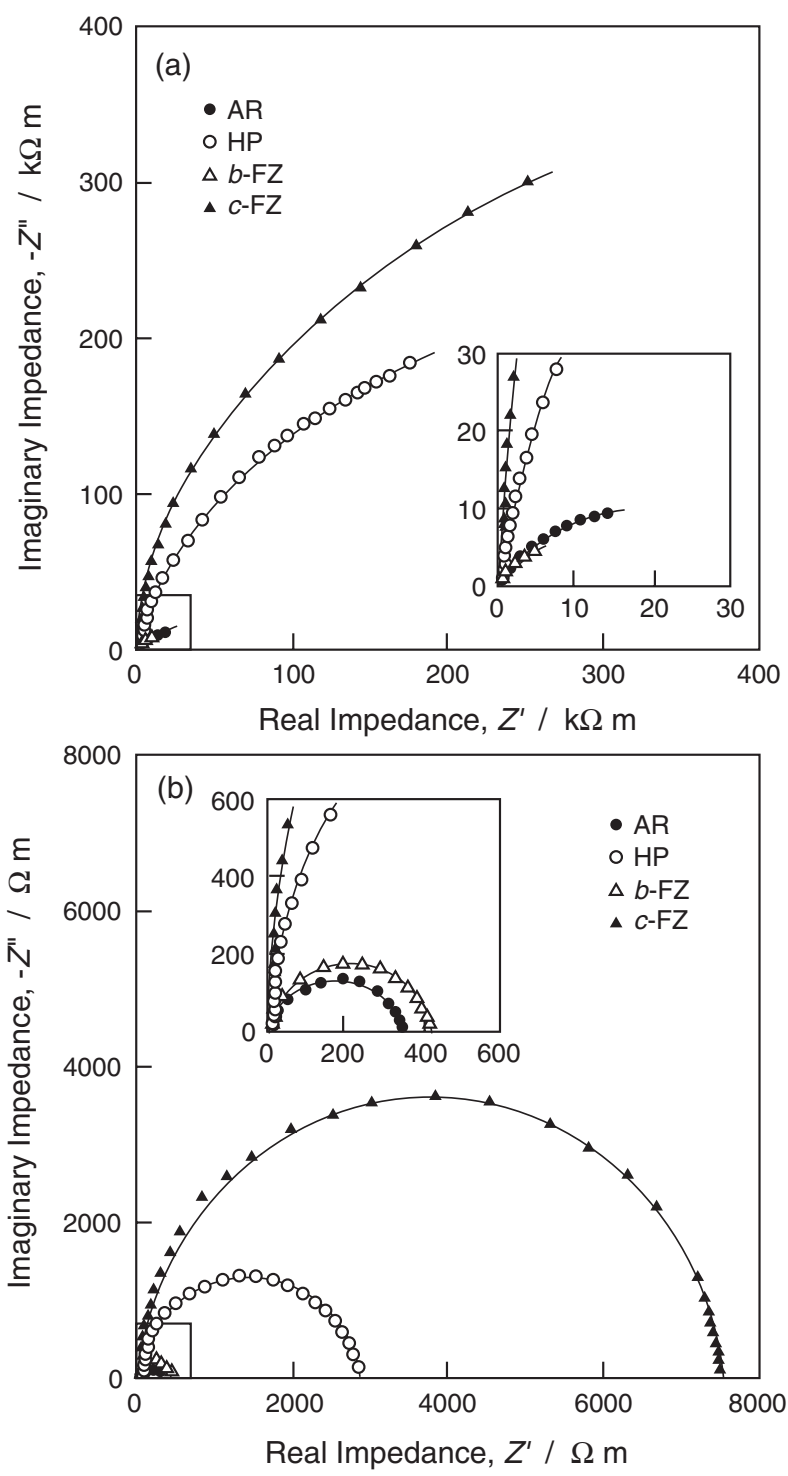

Fig. 3 Complex impedance plots at $T=$ (a) 743 and (b) $973 \mathrm{~K}$.

Figure 4 demonstrates the effect of frequency on the imaginary part of complex impedance $\left(Z^{\prime \prime}\right)$ and modulus $\left(M^{\prime \prime}\right)$ at $1023 \mathrm{~K}$. The $Z^{\prime \prime}$ and $M^{\prime \prime}$ of all specimens showed a 

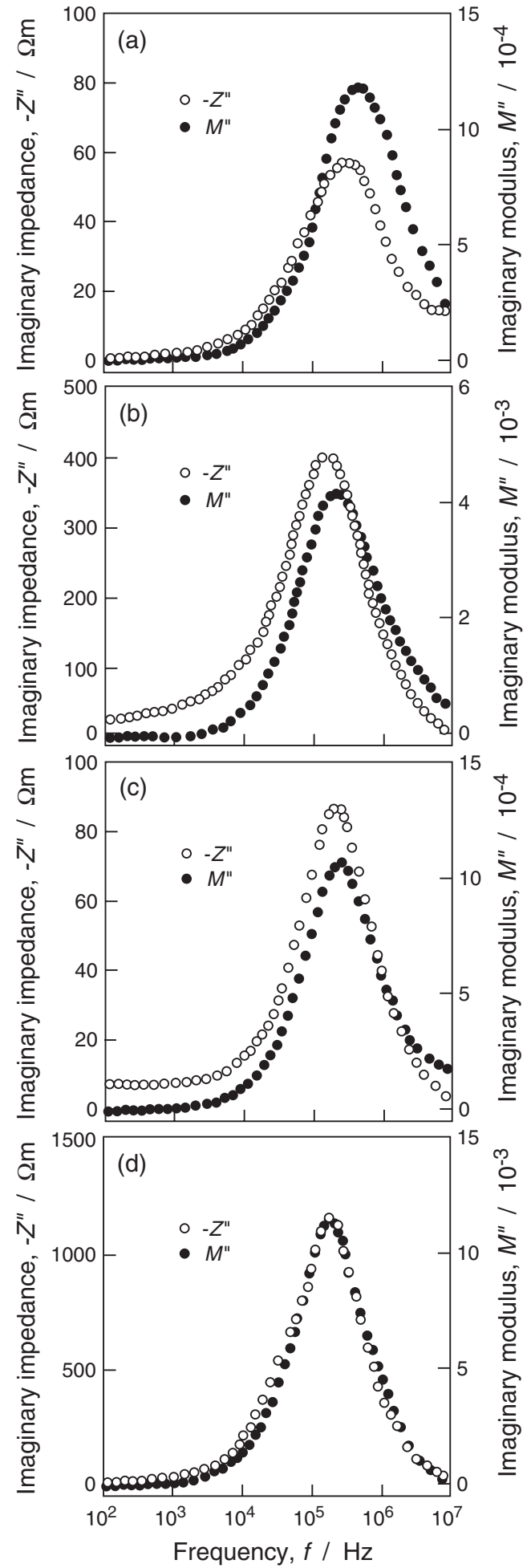

Fig. 4 Effect of frequency on imaginary impedance and modulus at $1023 \mathrm{~K}$. (a) AR, (b) HP, (c) $b$-FZ and (d) $c$-FZ.

single peak, implying the bulk response. Beltrán et al. synthesized $\mathrm{BT}_{2}$ powder by a sol-gel method, and sintered the $\mathrm{BT}_{2}$ powder at $1373 \mathrm{~K}$. They reported a single peak in $Z^{\prime \prime}$ and $M^{\prime \prime}$ vs. $f$ relationships, implying the single bulk response. ${ }^{9)}$ No relaxation process from grain boundary or surface was reported, which is in agreement with that of present study. The peak frequency for $Z^{\prime \prime}\left(f_{Z^{\prime \prime}}\right)$ should be theoretically coincident with that for $M^{\prime \prime}\left(f_{M^{\prime \prime}}\right){ }^{12)}$ However,

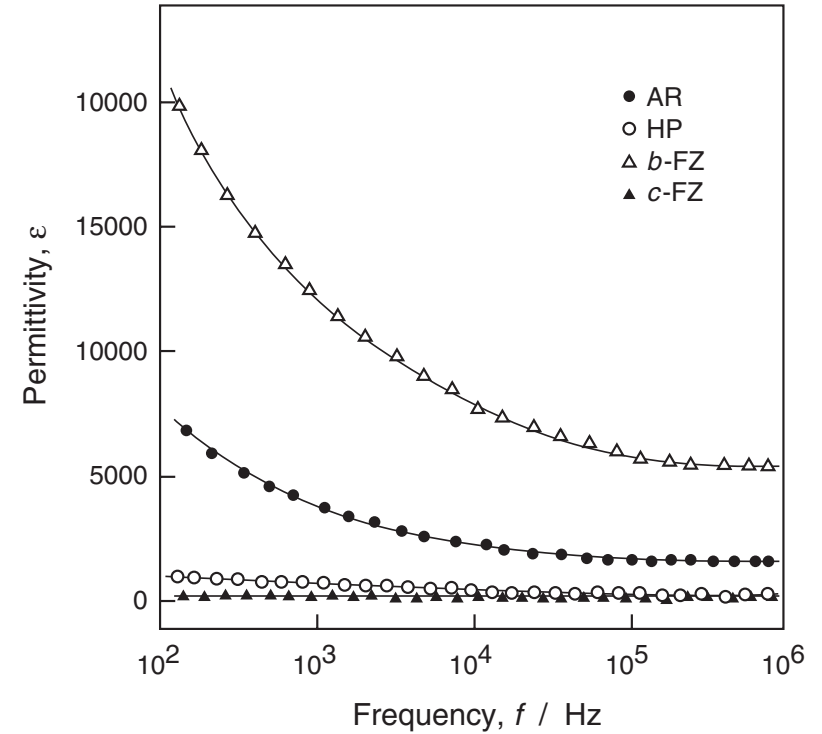

Fig. 5 Relationship between permittivity of and frequency at $T=743 \mathrm{~K}$.

the $f_{Z^{\prime \prime}}$ often differs from the $f_{M^{\prime \prime}}$ resulting from the defect structure or imhomogeneity of specimens. Beltrán et al. reported the significant deference between $f_{Z^{\prime \prime}}$ and $f_{M^{\prime \prime}}$ at high temperatures due to the partial decomposition of $\mathrm{BT}_{2} \cdot{ }^{9)} \mathrm{On}$ the other hand, the $f_{Z^{\prime \prime}}$ was almost in agreement to $f_{M^{\prime \prime}}$ in this study, suggesting homogeneous microstructure and no decomposition in the present $\mathrm{BT}_{2}$ specimens. In the $c$-FZ specimen, the $f_{Z^{\prime \prime}}$ exactly agreed with the $f_{M^{\prime \prime}}$ implying a sharp distribution of relaxation time associating with high crystallinity and excellent $c$-orientation. The $f_{Z^{\prime \prime}}$ of AR and $b$-FZ specimens slightly shifted from the $f_{M^{\prime \prime}}$, suggesting a slight disordering of crystal orientation. The difference between $f_{Z^{\prime \prime}}$ and $f_{M^{\prime \prime}}$ for the HP specimen was smaller than that of poly-crystalline $\mathrm{BT}_{2}$ sintered at 1473 by Beltrán et al. ${ }^{9)}$ This suggests that the HP specimen prepared by the powder from arc-melted buttons had higher thermal stability than that prepared by the sol-gel method.

Figure 5 depicts the frequency dependence of permittivity $(\varepsilon)$ at $743 \mathrm{~K}$. The permittivity decreased with increasing frequency and became almost constant over $100 \mathrm{kHz}$. Since the apparent permittivity would decrease with increasing frequency due to the resistivity component $(R),{ }^{13)}$ the true permittivity should be evaluated at a high frequency. Fig. 5 implies that the true permittivity can be obtained over $100 \mathrm{kHz}$. If the complex impedance plot had a full semicircle, the true permittivity can be obtained from the relationship of $\omega \tau=1$ at the top of semicircle. ${ }^{14)}$ If the resistivity is too high to obtain the full semicircle of the complex impedance plot, the true permittivity can be obtained from the frequency dependence of permittivity at the high frequency.

Figure 6 demonstrates the temperature dependence of permittivity for all specimens at $100 \mathrm{kHz}$. All specimens showed peaks around $750 \mathrm{~K}$ implying the ferroelectric to paraelectric transition. Although the $b$-axis orientation of AR specimen was primarily similar to that of $b$-FZ specimen, the maximum permittivity of AR specimen was about 2000 which was smaller than that of $b$-FZ $(\sim 6000)$. The smaller permittivity of AR specimens could be associated with the 


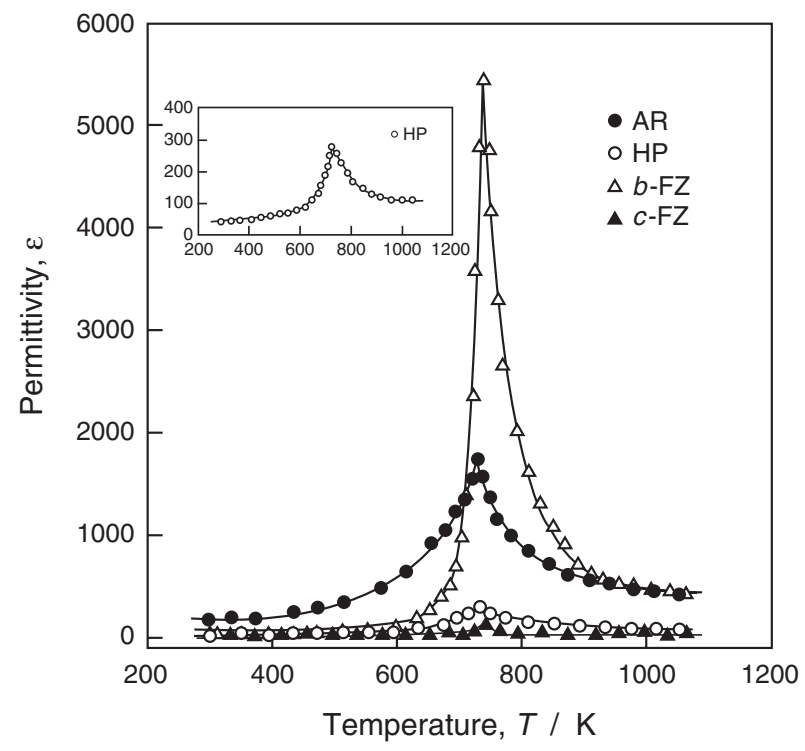

Fig. 6 Temperature dependence of permittivity for poly- and singlecrystalline $\mathrm{BT}_{2}$ at $100 \mathrm{kHz}$.

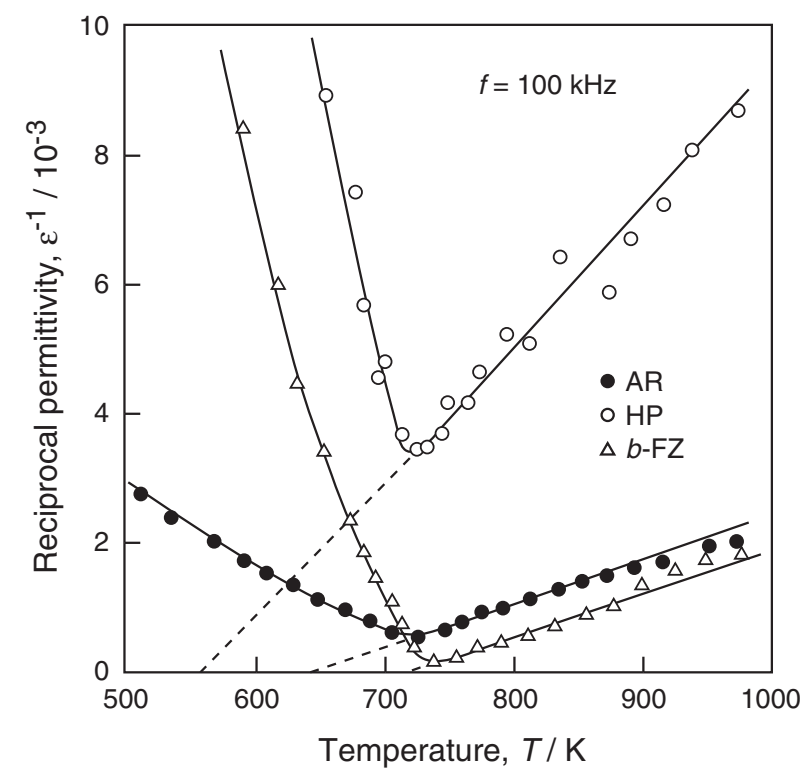

Fig. 7 Relationship between reciprocal permittivity and temperature for poly- and single-crystalline $\mathrm{BT}_{2}$ at $100 \mathrm{kHz}$.

crystal defects such as oxide vacancy formed during the rapid quenching in Ar atmosphere. The high-temperature annealing in air may also affect the decrease of permittivity because the decomposition of $\mathrm{BT}_{2}$ may locally proceed in grains. The HP specimen showed a peak $(\varepsilon=300)$ around $720 \mathrm{~K}$ resulting from the slight $b$-orientation as shown in Fig. 2(a). A small peak $(\varepsilon=50)$ was observed at $750 \mathrm{~K}$ for the $c$-FZ specimen. This may be caused of slight imperfection of $c$-orientation or small ferroelectricity in the $c$-direction.

Figure 7 demonstrates the relationship between the reciprocal permittivity $\left(\varepsilon^{-1}\right)$ and temperature. It is generally known that the $\varepsilon^{-1}$ commonly changes with temperature in many ferroelectric materials as given by eq. (1) over the Curie temperature $\left(T_{\mathrm{c}}\right){ }^{15)}$

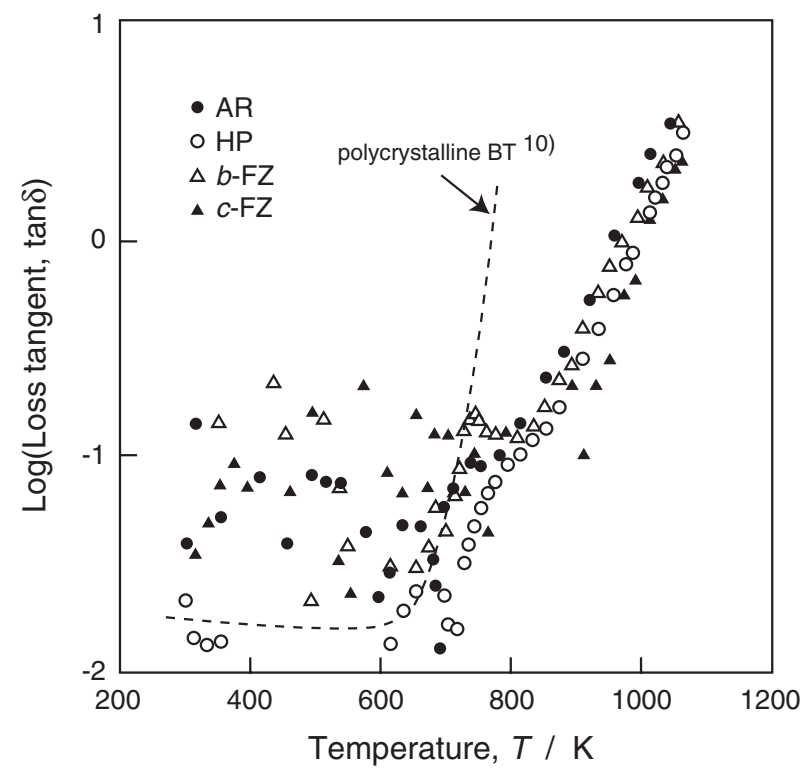

Fig. 8 Temperature dependence of loss tangent for poly- and singlecrystalline $\mathrm{BT}_{2}$ at $100 \mathrm{kHz}$.

$$
\varepsilon^{-1}=C^{-1} \cdot\left(T-T_{0}\right)
$$

where $C$ is a constant, and $T_{0}$ is called as Curie-Weiss temperature. The $T_{0}$ values of AR, HP and $b$-FZ specimens were 642,554 and $718 \mathrm{~K}$, respectively. The $C$ values were $1.52 \times 10^{5}, \quad 4.86 \times 10^{4}$ and $1.53 \times 10^{5} \mathrm{~K}$, respectively. Akishige et al. have reported that the $T_{0}$ and $C$ of singlecrystalline $\mathrm{BT}_{2}$ prepared by a flux method was $692 \mathrm{~K}$ and $3 \times 10^{5} \mathrm{~K}$, respectively. ${ }^{8)}$ These values were close to those of $b$-FZ specimens. Although the transition behavior around $T_{\mathrm{c}}$ for $\mathrm{BT}_{2}$ has been scarcely reported, BT has been widely investigated due to its wide applications. Abdelkefi et al. reported that the $T_{0}$ and $T_{\mathrm{c}}$ of poly-crystalline BT was 365 and $395 \mathrm{~K}$, respectively. ${ }^{16)}$ The difference between $T_{\mathrm{c}}$ and $T_{0}$ of poly-crystalline BT was about $30 \mathrm{~K}$. Drougard et al. reported that the $T_{0}$ and $T_{\mathrm{c}}$ of single-crystalline BT was 385 and $395 \mathrm{~K}$, respectively. ${ }^{17)}$ It is commonly understood that the $T_{0}$ is always lower than $T_{\mathrm{c}}$, typically $10 \mathrm{~K}$ for reliable specimens of BT, which suggests the first order transition. ${ }^{18)}$ In the present study, the difference of $T_{\mathrm{c}}$ and $T_{0}$ for $\mathrm{BT}_{2}$ specimens ranged from 15 to $169 \mathrm{~K}$. The smallest difference for $\mathrm{BT}_{2}$ was almost the same as that of BT. The phase transition mechanism of $\mathrm{BT}_{2}$ has been studied by Yashima et al., and reported as $C 2$ to $C 2 / m$ transition likely to be the first order transition. ${ }^{19)}$

Figure 8 shows the temperature dependence of dielectric loss $(\tan \delta)$ for all specimens at $100 \mathrm{kHz}$. The $\tan \delta$ slightly decreased or almost constant with increasing temperature up to around the $T_{\mathrm{c}}$, and then increased significantly. The $\tan \delta$ of AR specimen was highest among the all specimens. This suggests that the AR specimen has more defects sensitive to the ac field than the $b$-FZ specimen. The significant increase in the $\tan \delta$ over $T_{\mathrm{c}}$ could be contributed from the DC loss. ${ }^{20)}$ In Fig. 8, the $\tan \delta$ of BT was compared with that of $\mathrm{BT}_{2}$. The BT had low $\tan \delta$ values (smaller than 0.02) in a temperature range of 300 to $500 \mathrm{~K}$; however, it increased significantly with increasing temperature above $600 \mathrm{~K}^{10)}$ 
Temperature, $T$ / $\mathrm{K}$

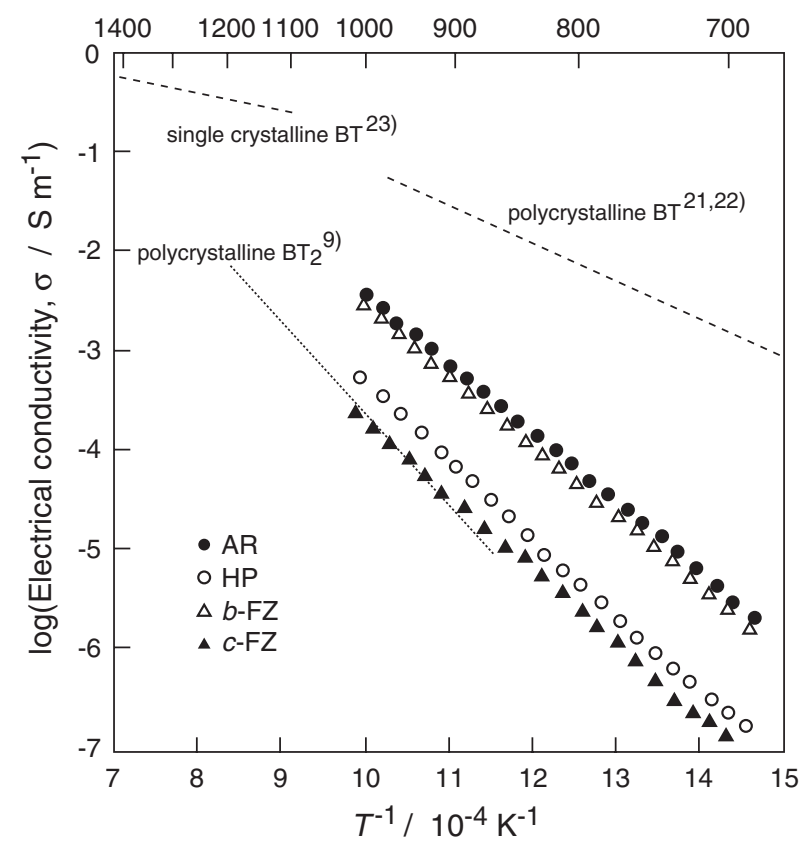

Fig. 9 Temperature dependence of electrical conductivity of poly- and single-crystalline $\mathrm{BT}_{2}$.

Therefore, the $\mathrm{BT}_{2}$ could be advantageous for applications due to its high $\varepsilon$ and low $\tan \delta$ at high-temperatures around 600 to $900 \mathrm{~K}$.

Figure 9 shows the temperature dependence of electrical conductivity $(\sigma)$ of the $\mathrm{BT}_{2}$ specimens comparing with polyand single-crystalline BT. The electrical conductivities of AR and $b$-FZ specimens were almost the same, and about 10 times as high as that of $c$-FZ specimens. The $\sigma$ of $b$-FZ and AR was 2 orders lower than that of poly- ${ }^{21,22)}$ and singlecrystalline BT. ${ }^{23)}$ The HP specimen had intermediate values between $b$-FZ and $c$-FZ specimens. The crystal structure of $\mathrm{BT}_{2}$ can be drawn by the combination of corner- and edgeshared $\mathrm{TiO}_{6}$ octahedrons. ${ }^{24)}$ The arrangement of $\mathrm{TiO}_{6}$ octahedrons is highly anisotropic. The connection of $\mathrm{TiO}_{6}$ octahedrons is rather staggered in the $a$ - and $c$-directions, and is well ordered in the $b$-direction as depicted in Figs. 10 (a) and (b), respectively. The ordering of $\mathrm{TiO}_{6}$ octahedrons might associated with the anisotropy of the electrical conductivity of $\mathrm{BT}_{2}$. The difference of $\sigma$ between poly- and single-crystalline for $\mathrm{BT}_{2}$ was greater than that for $\mathrm{BT}$. This could be caused of the greater crystallographic anisotropy of $\mathrm{BT}_{2}$ than that of BT.

\section{Conclusions}

Non-oriented and (010) oriented poly-crystalline $\mathrm{BT}_{2}$ were prepared by hot-pressing and arc-melting, respectively. The maximum permittivity of non-oriented and (010) oriented poly-crystalline $\mathrm{BT}_{2}$ was 300 and 2000 at $720 \mathrm{~K}$, respectively, whereas that of $\mathrm{BT}_{2}$ single-crystalline parallel to $(010)$ and $(001)$ was 6000 and 50 at the $T_{\mathrm{c}}(750 \mathrm{~K})$. The $\tan \delta$ of all specimens were between 0.01 and 0.2 below $T_{\mathrm{c}}$ and significantly increased with increasing temperature above $T_{\mathrm{c}}$. The electrical conductivity of (010) oriented (a)

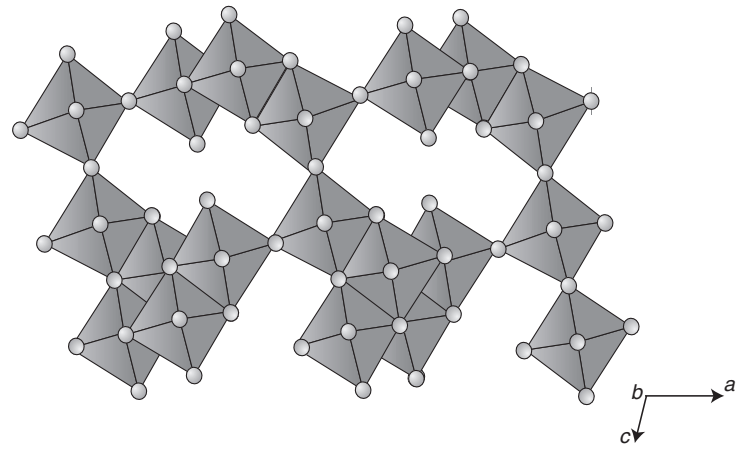

(b)

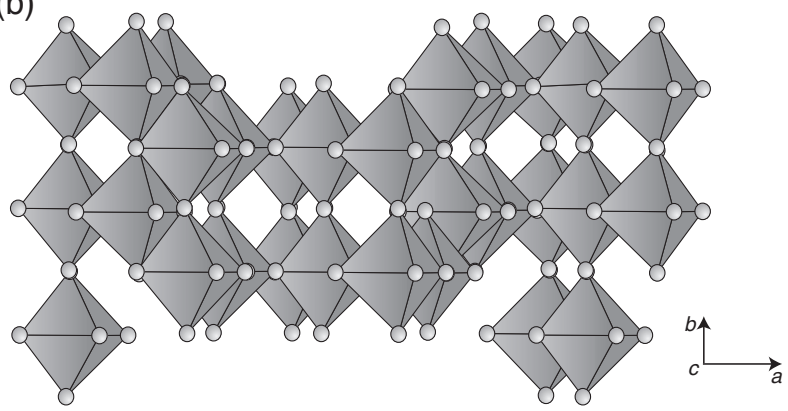

Fig. 10 Configuration of $\mathrm{TiO}_{6}$ octahedrons in (a) $a c$ and (b) $a b$ planes.

poly- and single-crystalline $\mathrm{BT}_{2}$ had almost same values, which were one order higher than that of non-oriented poly-crystalline $\mathrm{BT}_{2}$ and (001) oriented single-crystalline $\mathrm{BT}_{2}$.

\section{Acknowledgements}

The study was supported partly by the Grant-in-Aids for Exploratory Research (17656209) and the Scientific Research of Priority Areas (440-17042008) of the Ministry of Education, Culture, Sports, Science and Technology (MEXT), and the Asian CORE Program of the Japan Society for the Promotion of Science (JSPS).

\section{REFERENCES}

1) H. M. O 'Bryan, Jr. and J. Thomson, Jr.: J. Am. Ceram. Soc. 57 (1974) $522-526$.

2) D. E. Rase and R. Roy: J. Am. Ceram. Soc. 38 (1955) 102-113.

3) T. Negas, R. S. Roth, H. S. Parker and D. Minor: J. Solid State Chem. 9 (1974) 297-307.

4) J. J. Ritter, R. S. Roth and J. E. Blendell: J. Am. Ceram. Soc. 69 (1986) $155-162$.

5) K. W. Kirby and B. A. Wechsler: J. Am. Ceram. Soc. 74 (1991) 18411847.

6) T. Akashi, H. Iwata and T. Goto: Mater. Trans. 44 (2003) 1644-1646.

7) T. Akashi, H. Iwata and T. Goto: Mater. Trans. 44 (2003) 802-804.

8) Y. Akishige, K. Fukano and H. Shigematsu: Jpn. J. Appl. Phys. 42 (2003) L946-L948.

9) H. Beltrán, B. Gómez, N. Masó, E. Cordoncillo, P. Escribano and A. R. West: J. Appl. Phys. 97 (2005) 084104.

10) Y. Akishige and H. Shigematsu: J. Korean Phys. Soc. 46 (2005) 24-28.

11) J. T. S. Irvine, D. C. Sinclair and A. R. West: Adv. Mater. 2 (1990) 132-138.

12) A. R. West, D. C. Sinclair and N. Hirose: J. Electroceramics 1 (1997) 65-71.

13) W. Preis, A. Burgermeister, W. Sitte and P. Supancic: Solid State 
Ionics 173 (2004) 69-75

14) D. C. Sinclair and A. R. West: J. Appl. Phys. 66 (1989) 3850-3856.

15) C. Kittel: Introduction to Solid State Physics, pp. 467-483.

16) H. Abdelkefi, H. Khemakhem, G. Vélu, J. C. Carru and R. Von der Mühll: J. Alloys Compounds 399 (2005) 1-6.

17) M. E. Drougard and D. R. Young: Phys. Rev. 95 (1954) 1152-1153.

18) D. Richter and S. Trolier-McKinstry: Nanoelectronics and Information Technology, (Wiley-VCH, Weinheim, 2003) p. 59.

19) M. Yashima, R. Tu, T. Goto and H. Yamane: Appl. Phys. Lett. 87
(2005) 101909.

20) Y. Wu, M. J. Forbess, S. Seraji, S. J. Limmer, T. P. Chou and G. Cao: J. Appl. Phys. 89 (2001) 5647-5652.

21) T. Akashi, K. Morita, T. Hirai, H. Yamane and T. Goto: Mater. Trans. 42 (2001) 1823-1826.

22) N. Hirose and A. R. West: J. Am. Ceram. Soc. 79 (1996) 1633-1641.

23) J. Nowotny and M. Rekas: Ceramics International 20 (1994) 225-235.

24) T. Kimura, T. Goto, H. Yamane, H. Iwata, T. Kajiwara and T. Akashi: Acta Cryst. C 59 (2003) i128-130. 